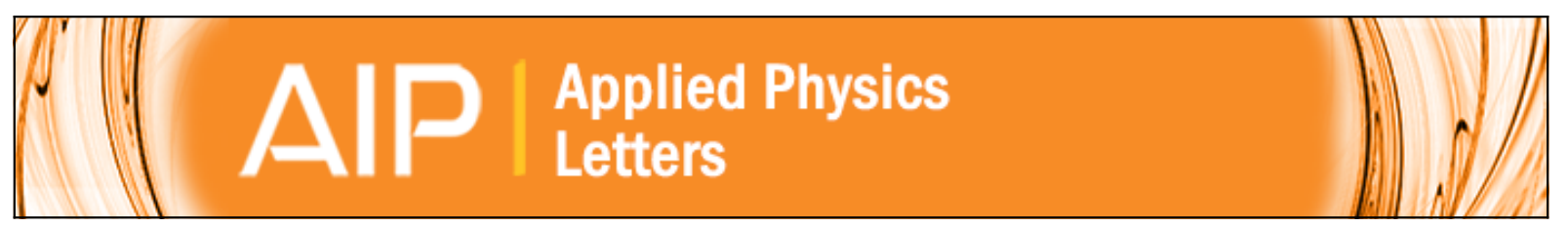

\title{
Excitation power dependence of the Purcell effect in photonic crystal microcavity lasers with quantum wires
}

J. Canet-Ferrer, I. Prieto, G. Muñoz-Matutano, L. J. Martínez, L. E. Muñoz-Camuniez, J. M. Llorens, D. Fuster, B. Alén, Y. González, L. González, P. A. Postigo, and J. P. Martínez-Pastor

Citation: Applied Physics Letters 102, 201105 (2013); doi: 10.1063/1.4807439

View online: http://dx.doi.org/10.1063/1.4807439

View Table of Contents: http://scitation.aip.org/content/aip/journal/apl/102/20?ver=pdfcov

Published by the AIP Publishing

\section{Articles you may be interested in}

Heterogeneous integration and precise alignment of InP-based photonic crystal lasers to complementary metaloxide semiconductor fabricated silicon-on-insulator wire waveguides

J. Appl. Phys. 107, 063103 (2010); 10.1063/1.3319667

Coupled optoelectronic modeling and simulation of nanowire lasers

J. Appl. Phys. 100, 044305 (2006); 10.1063/1.2221514

Surface-emitting microlaser combining two-dimensional photonic crystal membrane and vertical Bragg mirror Appl. Phys. Lett. 88, 081113 (2006); 10.1063/1.2172730

Quasiperiodic photonic crystal microcavity lasers

Appl. Phys. Lett. 84, 4875 (2004); 10.1063/1.1762705

Experimental demonstration of a high quality factor photonic crystal microcavity

Appl. Phys. Lett. 83, 1915 (2003); 10.1063/1.1606866

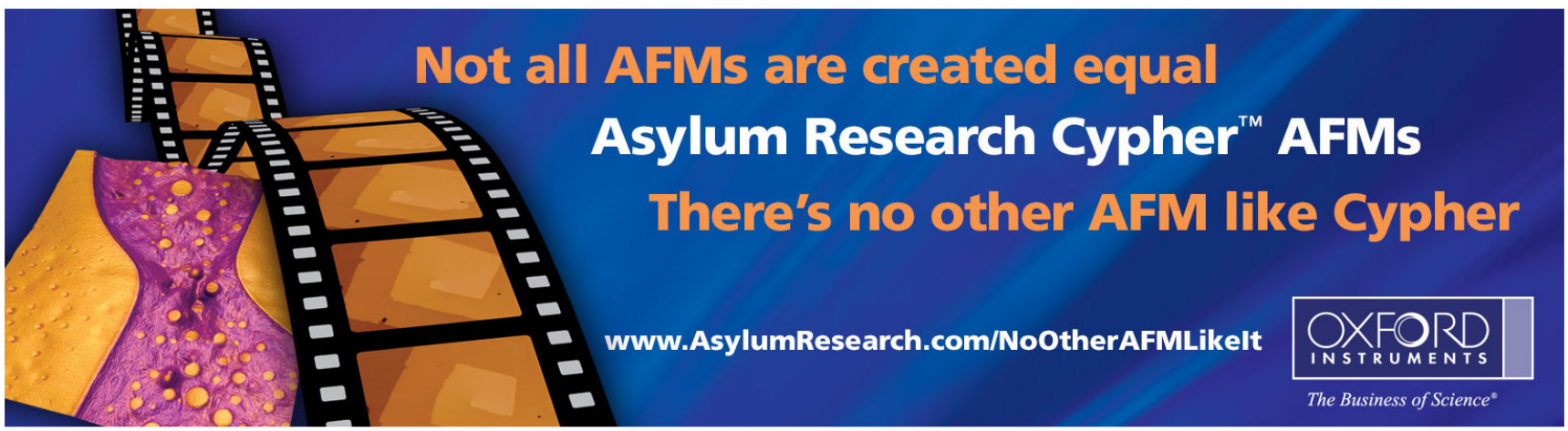




\title{
Excitation power dependence of the Purcell effect in photonic crystal microcavity lasers with quantum wires
}

\author{
J. Canet-Ferrer, ${ }^{1, a)}$ I. Prieto, ${ }^{2}$ G. Muñoz-Matutano, ${ }^{1}$ L. J. Martínez, ${ }^{2}$ \\ L. E. Muñoz-Camuniez, ${ }^{2}$ J. M. Llorens, ${ }^{2}$ D. Fuster, ${ }^{2}$ B. Alén, ${ }^{2}$ Y. González, ${ }^{2}$ \\ L. González, ${ }^{2}$ P. A. Postigo, ${ }^{2}$ and J. P. Martínez-Pastor ${ }^{1}$ \\ ${ }^{1}$ Institut de Ciència dels Materials, Universitat de València, P.O. Box 22085, 46071 Valencia, Spain \\ ${ }^{2}$ IMM-Instituto de Microelectrónica de Madrid (CNM-CSIC), Isaac Newton 8, PTM, E-28760 Tres Cantos, \\ Madrid, Spain
}

(Received 14 December 2012; accepted 4 May 2013; published online 21 May 2013)

\begin{abstract}
The Purcell effect dependence on the excitation power is studied in photonic crystal microcavity lasers embedding InAs/InP quantum wires. In the case of non-lasing modes, the Purcell effect has low dependence on the optical pumping, attributable to an exciton dynamics combining free and localized excitons. In the case of lasing modes, the influence of the stimulated emission makes ambiguous the determination of the Purcell factor. We have found that this ambiguity can be avoided by measuring the dependence of the decay time on the excitation power. These results provide insights in the determination of the Purcell factor in microcavity lasers. (C) 2013 AIP Publishing LLC.

[http://dx.doi.org/10.1063/1.4807439]
\end{abstract}

The Purcell effect (PE) has been widely demonstrated on systems combining photonic microcavities with embedded quantum nanostructures ${ }^{1-3}$ to enhance the spontaneous emission (SE) rate. ${ }^{4-7}$ The PE is also relevant for fast modulation purposes $^{8}$ and efficient laser emission. ${ }^{9-11}$ The Purcell factor (PF) accounts for the interaction between a quantum emitter and the optical cavity mode. Under ideal conditions of spectral, spatial, and polarization matching between the emitter and the mode, the magnitude of such interaction equals the figure of merit, $F_{p} \cdot{ }^{12}$ In the case of an optical mode in a photonic crystal microcavity (PCM) a high $F_{p}$ is provided due to the large quality factor to mode volume ratio $(Q / V) .{ }^{1}$ Onedimensional nanostructure emitters like quantum wires (QWRs) are of great interest for laser devices since they exhibit a reduced dimensionality and a large density of states. $^{13,14}$ In addition, the nanostructures based on InAs/InP enable the fabrication of light sources at the spectral windows of interest from the technological point of view (1.31 and $1.55 \mu \mathrm{m}) .{ }^{15,16}$ Since the early demonstration of lasing action in QWR-based devices, ${ }^{17}$ further improvements have been made in the performance of the host optical cavities. PCMs have provided low threshold powers in QWR lasers at $\operatorname{low}^{18}$ and room temperature. ${ }^{19}$ To understand in deep the laser emission and for an accurate treatment of the rate equation formalism in microcavity lasers, it is necessary to determine the PF. ${ }^{10,20}$ The correct and unambiguous determination of the PF may also provide insights in the determination of the laser threshold. ${ }^{21}$

In this work we have measured the dependence of the decay time on the excitation power in lasing and non-lasing optical modes of PCMs with QWRs by time resolved measurements. We have found that in non-lasing modes the decay time varies softly with the power due to the increasing contribution of free excitons. In lasing modes it has been measured an expected decrease of the decay time as the excitation power increases. The decrease takes place even at very low

a)Jose.Canet-Ferrer@uv.es excitation powers, making ambiguous the determination of the PF value, which might be easily overestimated. We have found that in this situation the PF can still be determined by measuring the variation of the time decay with the excitation power.

Photonic crystal L7-microcavities ${ }^{22}$ fabricated on InP membranes and containing a single layer of InAs selfassembled QWRs emitting at $1.5 \mu \mathrm{m}$ have been fabricated. The self-assembled InAs/InP QWRs were grown by solid source molecular beam epitaxy. This active layer was embedded into a $237 \mathrm{~nm}$ slab of InP deposited onto a $929 \mathrm{~nm}$ thick sacrificial layer of $\operatorname{In}_{0.53} \mathrm{Ga}_{0.47}$ As. More details about growth, morphology, and low temperature emission properties of the QWRs can be found in Ref. 15. The L7-type PCMs were fabricated by removing seven holes along the $\Gamma-\mathrm{K}$ direction of a triangular lattice. ${ }^{23}$ The lattice constant was $a=410 \mathrm{~nm}$ while the radius $(r)$ of the holes was changed from $0.20 a$ to $0.32 a$ to tune the spectral position of the fundamental mode with the emission of the QWRs. The linear defect of the cavity is oriented parallel to the [1-10] direction of the InP substrate, i.e., parallel to the QWRs. Finitedifference time-domain (FDTD) calculations show that Q-values are within $2 \times 10^{4}$ and $2 \times 10^{5}$, while the mode volume and effective index $\left(n_{\text {eff }}\right)$ for the fundamental mode are $1.1(\lambda / \mathrm{n})^{3}$ and 2.78 , respectively. ${ }^{19}$ The QWRs exhibit a broad emission and optical absorption, spatial, and spectral detuning resulting in a measured PF not larger than $3 .^{10,24,25}$ This value of PF provides a weak coupling regime at the same time than lasing, which makes our system especially suitable for PE studies on lasing modes.

For the measurement of the PF we have used time resolved micro-photoluminescence spectroscopy (TRPL) at $77 \mathrm{~K}$ using a fiber based confocal microscope (effective diaphragm aperture $\approx 5 \mu \mathrm{m}$ ). Excitation was done at $980 \mathrm{~nm}$ (below the InP absorption band edge) by a ps-pulsed laser diode with a repetition rate of $40 \mathrm{MHz}$. An aspheric lens $(\mathrm{NA}=0.6)$ was employed to focus the collection and the excitation light under confocal conditions with a spot 

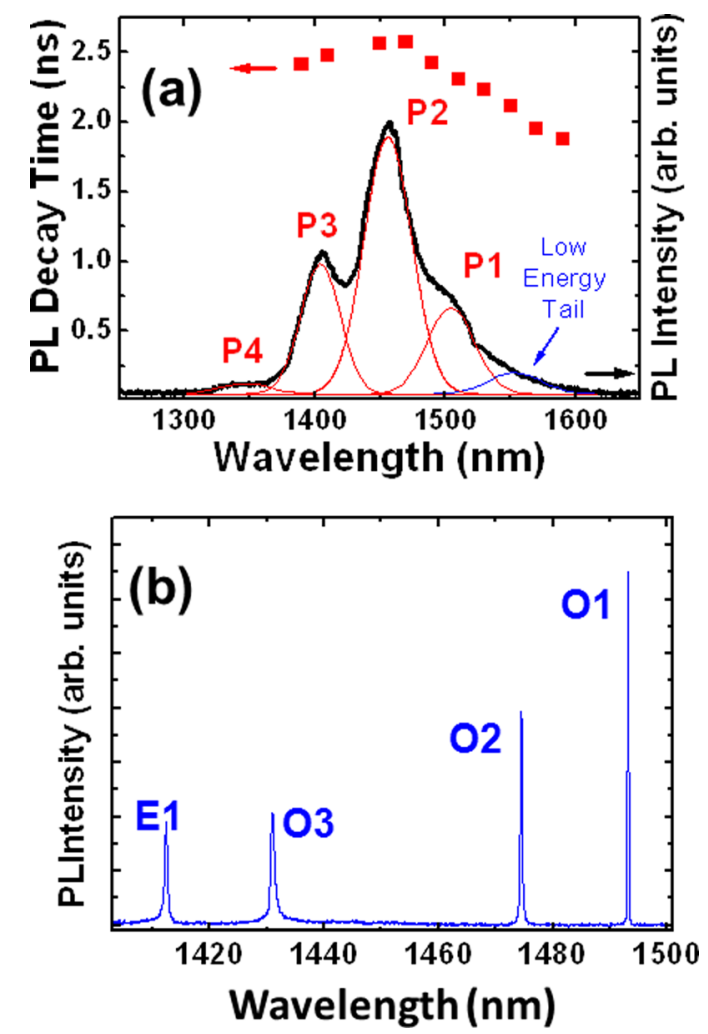

FIG. 1. (a) PL spectrum and lifetime at $77 \mathrm{~K}$ of QWRs ensemble outside of the PCMs. (b) PL spectrum of an L7-PCM with QWRs showing the optical modes. The fundamental mode is labeled as $\mathrm{O} 1$.

diameter $\approx 1.5 \mu \mathrm{m}$. The collected light was guided to a $0.5 \mathrm{~m}$ focal length spectrometer and detected by an InGaAs linear photodetector array. TRPL spectra were measured using an InGaAs avalanche photo-diode. The PF is determined by measuring the decay time of the emitters coupled to an optical cavity mode $\left(\tau_{\mathrm{CAV}}\right)$ with respect to the decay time of the bare emitters located outside of the cavity $\left(\tau_{0}\right)$.
Figure 1(a) shows the PL spectrum of an ensemble of bare QWRs at $77 \mathrm{~K}$. It consists of a broad band centered at $1451 \mathrm{~nm}$, which is fitted as the sum of several Gaussian peaks, each of them ascribed to QWRs of different height. ${ }^{16}$ The QWRs present slightly different exciton lifetimes, ranging from 1.8 to $2.7 \mathrm{~ns}$ at the wavelengths of interest. Figure 1(b) shows the PL spectrum corresponding to the mode structure of a L7-PCM with QWRs. Four narrow peaks can be observed, corresponding to the lowest-energy optical modes of the L7 PCM (labeled as O1, O2, O3, and E1). The QWRs within the PCM result in laser emission if spectral matching conditions are met.

Figure 2(a) shows the integrated laser emission intensity versus the excitation power (L-L curve) obtained in an L7PCM laser, with $a=410 \mathrm{~nm}$ and $r / a \approx 0.29$. The lasing occurs in the fundamental mode $\mathrm{O} 1$ at the wavelength $\lambda(\mathrm{O} 1)=1442 \mathrm{~nm}$ with $\mathrm{a}=15500$ at the low excitation power regime. The L-L curve shows a soft kink between the excitation power values $6 \mu \mathrm{W}$ and $12 \mu \mathrm{W}$, noted by the change in the slope. Such a soft transition from spontaneous to stimulated emission is characteristic of high beta lasers. ${ }^{26}$ The L-L curve was fitted using the stationary solution of a rate equations model. ${ }^{19} \mathrm{We}$ have considered as free parameters the spontaneous emission factor $\beta$, the differential gain $g$, and the transparency carrier density $N_{\text {tr }}$. The other parameters of the model were fixed as in Ref. 20 provided that the material, the type of cavity, and the fabrication process are the same. Only the cavity photon lifetime and the nonradiative recombination rate have been considered to take a different value. The photon lifetime is $12 \mathrm{ps}$ according to the $\mathrm{Q}$ factor of this cavity (15500). The non-radiative recombination processes are negligible at this temperature $(77 \mathrm{~K})$ for this type of QWRs as follows from the analysis performed in Ref. 15. The best fitting is obtained for $\beta=0.15$, $g_{0}=1.4 \times 10^{-14} \mathrm{~cm}^{2}$, and $N_{\text {tro }}=7.9 \times 10^{16} \mathrm{~cm}^{-3}$. However, the threshold pump power might be located in principle at
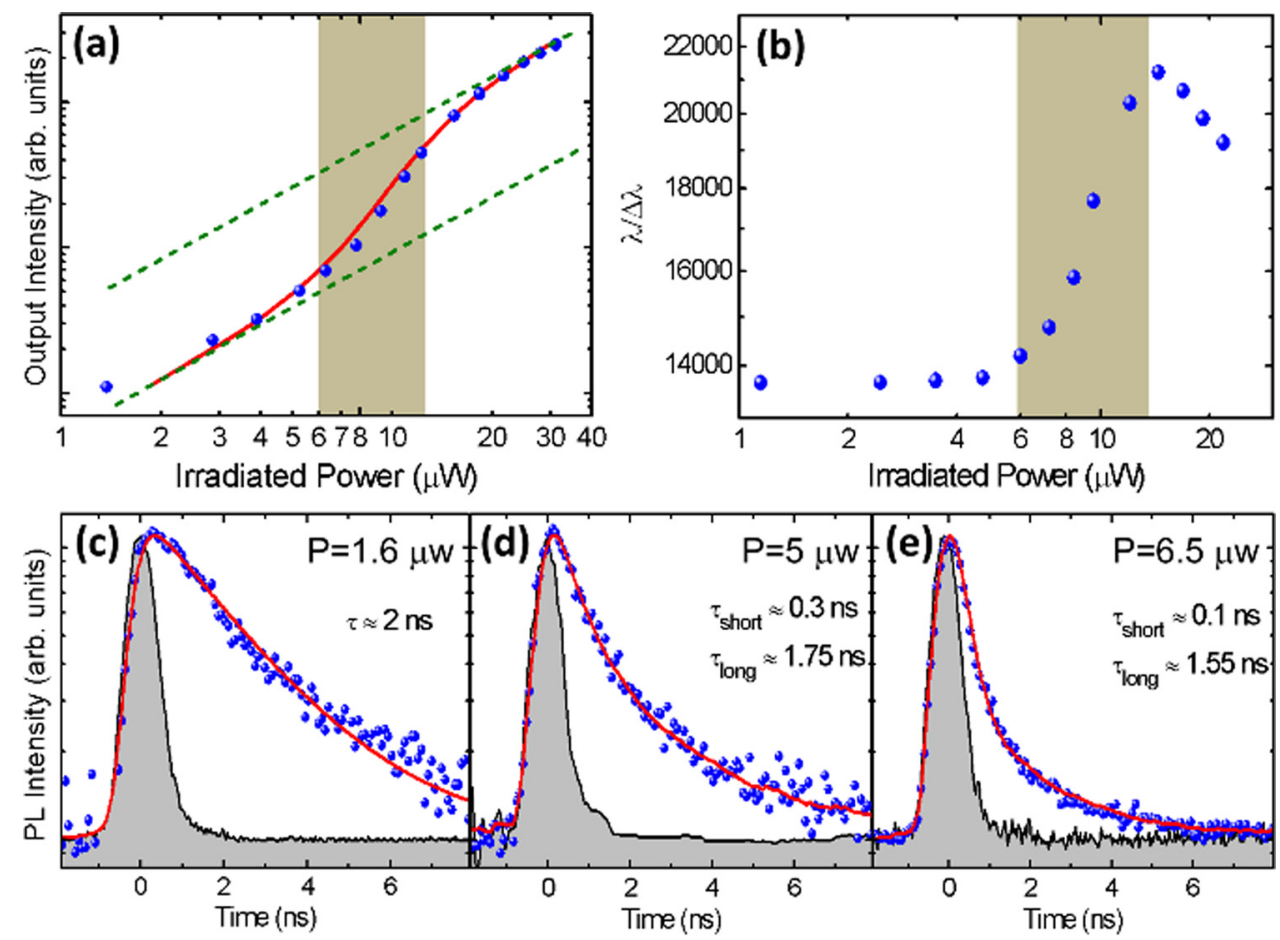

FIG. 2. (a) Integrated optical intensity of an L7-PCM laser embedding QWRs with the fitting to the rate equations (red solid line). (b) $\lambda / \Delta \lambda$ as a function of the excitation power. The threshold region is marked in grey. Panels (c)-(e) show the PL transients for three different values of incident power: $1.6 \mu \mathrm{W}$ (c), $5 \mu \mathrm{W}$ (d), and $6.5 \mu \mathrm{W}$ (e). System response (shaded grey), experimental data (dots), and fitting (solid line) against a single (c) or double (d) and (e) exponential decay function. 
any point covered by the soft kink region, between 6 and $12 \mu \mathrm{W}$. For those values, the fitting parameters $\left(\beta, g, N_{\mathrm{tr}}\right)$ obtained are $\left(0.1,0.67 g_{0}, 0.75 N_{\text {tro }}\right)$ at $P_{\text {th }}=6 \mu \mathrm{W}$ and $(0.3$, $\left.2.2 g_{0}, 1.5 N_{\text {tro }}\right)$ at $P_{\text {th }}=12 \mu \mathrm{W}$, which result in the same curve depicted by the solid line of Fig. 2(a). Hence, we conclude that our laser can be safely characterized within the $P_{\text {th }}$ values above mentioned. Figure 2(b) shows the evolution of the $\lambda / \Delta \lambda$ value, which is nearly constant $\approx 13500$ below $5.6 \mu \mathrm{W}$ and increases for larger excitation powers, reaching a maximum value $\approx 21000$ around $14.4 \mu \mathrm{W}$. Figures $2(\mathrm{c})-2(\mathrm{e})$ show the influence of the optical amplification in the PL transients measured at the wavelength of the $\mathrm{O} 1$ mode. At $1.6 \mu \mathrm{W}$ the PL transient is essentially mono-exponential with a decay time $\sim 2 \mathrm{~ns}$ [Fig. 2(c)]. When the optical pumping increases to $5 \mu \mathrm{W}$, the mono-exponential decay does not fit well and a double exponential function with a short and long decay times is required [Figs. 2(d) and 2(e)]. The short decay times are $0.3 \mathrm{~ns}$ at $4.8 \mu \mathrm{W}$ and $0.1 \mathrm{~ns}$ at $6.5 \mu \mathrm{W}$ (close to the system response). On the other hand, the long decay time presents a smaller dependence on the excitation power (within 1.75-1.55 ns). The existence of a long decay time can be attributed to the existence of photon recycling processes, as reported in H-type PCMs with an embedded InGaAsP QW. ${ }^{10}$ In our case, we also expect a background contribution from QWRs poorly coupled to the lasing mode of the PCM. ${ }^{24}$

To evaluate the influence of the stimulated emission in the dependence of the decay times on the excitation power, we have compared time resolved measurements of lasing and nonlasing modes from different PCMs at nearly equal wavelengths. Figure 3(a) shows the PL spectra of two PCMs with the same lattice constant $a=410 \mathrm{~nm}$, but with different radii, i.e., $r / a=0.22$ and 0.25 . The first cavity has $\lambda^{\mathrm{NL}}(\mathrm{O} 1)$ $=1516 \mathrm{~nm}$ and does not exhibit laser operation. The second cavity $(\mathrm{Q}=17300)$ presents laser operation on its fundamental mode at $\lambda^{\mathrm{L}}(\mathrm{O} 1)=1483 \mathrm{~nm}$. This mode was analyzed following the same method as for the laser mode in Fig. 2. In this case, the cavity photon lifetime is $14 \mathrm{ps,} \mathrm{slightly} \mathrm{higher} \mathrm{than} \mathrm{that} \mathrm{of}$ Fig. 2. As before, we have performed a series of fittings, in this case covering the range from $8 \mu \mathrm{W}$ to $20 \mu \mathrm{W}$. Within that range, the fitting parameters $\left(\beta, g, N_{\text {tr }}\right)$ take the values from $\left(0.026,0.47 g_{0}, 0.57 N_{\text {tro }}\right)$ to $\left(0.17,3.2 g_{0}, 1.4 N_{\text {tro }}\right)$, respectively. The values of $P_{\text {th }}$ are higher than those for the laser analyzed in Fig. 2 due to the lower gain, as derives from the fittings. This means that we have less QWRs coupled to the optical mode, which is consistent with the spectral tuning of that laser, situated between QWR families P1 and P2 shown in Fig. 1(a). ${ }^{15}$ The wavelength of the lasing mode, $\lambda^{\mathrm{L}}(\mathrm{O} 1)$, is spectrally close to that of the second mode of the non-lasing cavity, $\lambda^{\mathrm{NL}}(\mathrm{O} 2)=1490 \mathrm{~nm}$, which allows the comparison of the PF values obtained for each mode.

Figure 3(c) shows the dependence of the decay time on the excitation power for the ensemble of QWRs at $1516 \mathrm{~nm}$, which gives an increase of $\tau_{0}$ from 1.67 to $1.85 \mathrm{~ns}$ when the optical pumping intensity is reduced. This effect is due to the influence of the localized states. ${ }^{15}$ The same figure shows the evolution of the decay time for the non-lasing mode $\mathrm{O} 1$ at $\lambda^{\mathrm{NL}}(\mathrm{O} 1)=1516 \mathrm{~nm}$. Figure $3(\mathrm{~d})$ shows the evolution of the decay time for the non-lasing mode $\mathrm{O} 2$ at $\lambda^{\mathrm{NL}}(\mathrm{O} 2)=1490 \mathrm{~nm}$ and the influence of the localized states for the ensemble of QWRs at $1486 \mathrm{~nm}$, where a variation from 1.86 to $2.17 \mathrm{~ns}$ is measured for $\tau_{0}$. Using those values we can determine the $\mathrm{PF}=\tau_{0} / \tau_{\mathrm{CAV}}$ for the non-lasing modes at different excitation powers.

The PF of the $\mathrm{O} 1$ and $\mathrm{O} 2$ modes of the non-lasing cavity vary slightly from 1.24 and 1.29 , respectively, at the lowest excitation power, to 1.44 and 1.58 at high powers (see Table I). Figure 3(d) also shows the evolution of the time decay with the power for the lasing mode $\mathrm{O} 1$ at $\lambda^{\mathrm{L}}(\mathrm{O} 1)=1483 \mathrm{~nm}$. A double exponential function, as in Figs. 2(d) and 2(e), is
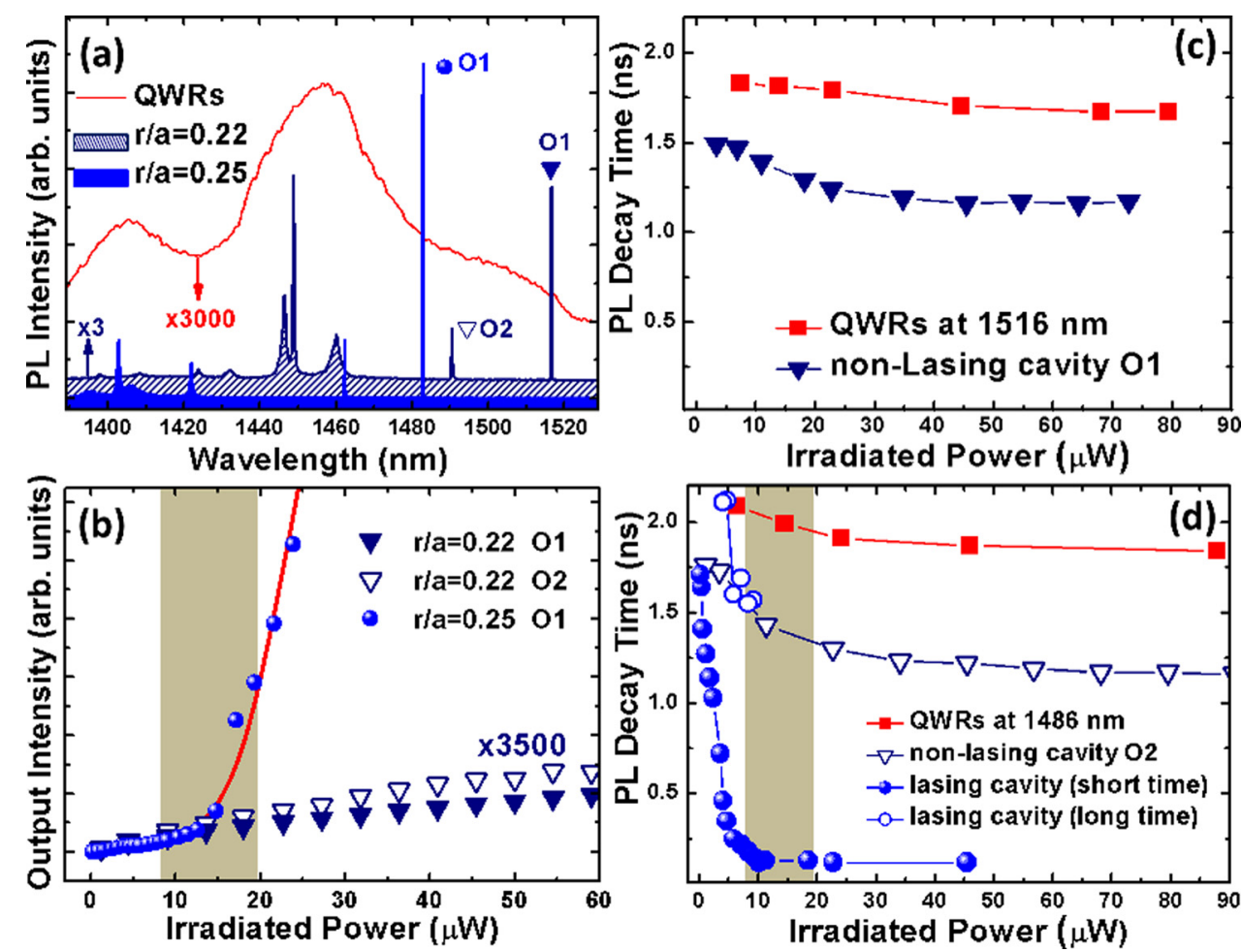

FIG. 3. (a) PL spectra of the bare QWRs (red line), lasing cavity $(r / a=0.22$, solid shadow), and a non-lasing cavity $(r / a=0.25$, light shadow). (b) Integrated optical intensity of the modes under study. The shadowed area determines the threshold region, and the red line is the fitting to the rate equations model. (c) Decay times versus excitation power of the $\mathrm{O} 1$ mode in the non-lasing cavity (solid triangles) to be compared with that of the QWRs emitting at $1516 \mathrm{~nm}$ wavelength (red squares). (d) Decay time versus excitation power of the $\mathrm{O} 2$ mode (empty triangles) in the non-lasing cavity and of the $\mathrm{O} 1$ mode in the lasing cavity (solid and empty circles for the short and large decay times, respectively). The decay time corresponding to QWRs emitting at $1486 \mathrm{~nm}$ is labeled with red squares. 
TABLE I. Spectral properties and decay times of QWRs and $\tau_{0} / \tau_{\mathrm{CAV}}$ for the studied modes at different excitation powers.

\begin{tabular}{|c|c|c|c|c|c|c|}
\hline & \multirow[b]{2}{*}{ Power $(\mu \mathrm{W})$} & \multicolumn{2}{|c|}{ Quantum wires } & \multicolumn{2}{|c|}{ Non-lasing cavity } & \multirow{2}{*}{$\frac{\text { Lasing cavity }}{\mathrm{O} 1}$} \\
\hline & & Family P1 & Family P2 & O1 & $\mathrm{O} 2$ & \\
\hline Wavelength $(\mathrm{nm})$ & & 1516 & 1486 & 1516 & 1490 & 1483 \\
\hline$\lambda / \Delta \lambda$ & 2.5 & $\ldots$ & $\ldots$ & 18500 & 7400 & 17200 \\
\hline \multirow[t]{3}{*}{$\tau(\mathrm{ns})$} & 0 & 1.85 & 2.17 & 1.51 & 1.80 & 1.80 \\
\hline & 7.5 & 1.83 & 2.09 & 1.47 & 1.62 & 0.33 \\
\hline & 70 & 1.67 & 1.86 & 1.16 & 1.17 & $\ldots$ \\
\hline \multirow[t]{3}{*}{$\tau_{0 /} \tau_{\mathrm{CAV}}$} & 0 & $\ldots$ & $\ldots$ & 1.23 & 1.20 & 1.20 \\
\hline & 7.5 & $\ldots$ & $\ldots$ & 1.24 & 1.29 & $\ldots$ \\
\hline & 70 & $\ldots$ & $\ldots$ & 1.44 & 1.58 & $\ldots$ \\
\hline
\end{tabular}

required to fit the PL transients. If we use the short decay time obtained from the fittings, the ratio $\tau_{0} / \tau_{\mathrm{CAV}}$ can take values above 4 even at excitation powers of $7 \mu \mathrm{W}$, below the kink region. Since the localization effects must be similar in both lasing and non-lasing modes, we must attribute the decrease of the short decay time component just to light amplification. Only when the excitation power is below $3 \mu \mathrm{W}$ the PL transients can be fitted to a single exponential. To safely obtain the PF value, we extrapolate to zero excitation power the evolution of the time decay with the excitation power of Fig. 3(d). We find a $\mathrm{PF}=1.2$ at zero excitation power, which is quite similar to that measured in the nonlasing modes. The PF values of the non-lasing modes are in agreement with experimental results and simulations ${ }^{15}$ for the same kind of cavities. The evolution of the PF with high excitation powers is explained by a reduction of the contribution of localization effects. In this case, the larger wavefunction extension of the free excitons (with respect to that of the localized ones) favors the overlap with the electromagnetic field of the cavity mode and hence the spatial tuning. ${ }^{15}$

In summary, we have investigated the Purcell effect in lasing and non-lasing L7-PCMs embedding self-assembled InAs/InP QWRs. The decay transients have been studied as a function of the excitation power. In non-lasing modes, we have found that the PF slightly increases with excitation power. We attribute this effect to the higher overlap between excitons and cavity modes as the emission of localization centers saturates. In lasing modes, the decay time is strongly influenced by the light amplification, even at very low excitation powers. For such modes we have estimated the PF at zero excitation power from the evolution of $\tau_{0} / \tau_{\mathrm{CAV}}$ with the excitation power.

We want to acknowledge financial support from the Spanish MICINN through grants (Nos. S-0505-TIC-0191, TEC2008-06756-C03-01/-03, TEC2011-29045-C04-03, TEC2011-29120-C05-01/04, and CAM S2009ESP-1503). J.C.-F. thanks the Spanish MCI for his FPI grant (No. BES2006-12300).
${ }^{1}$ J. M. Gérard, B. Sermage, B. Gayral, B. Legrand, E. Costard, and V. Thierry-Mieg, Phys. Rev. Lett. 81, 1110 (1998).

${ }^{2}$ D. Englund, D. Fattal, E. Waks, G. Solomon, B. Zhang, T. Nakaoka, Y. Arakawa, Y. Yamamoto, and J. Vuckovic, Phys. Rev. Lett. 95, 013904 (2005).

${ }^{3}$ M. Munsch, A. Mosset, A. Auffèves, S. Seidelin, J. P. Poizat, J. M. Gérard, A. Lemaître, I. Sagnes, and P. Senellart, Phys. Rev. B 80, 115312 (2009).

${ }^{4}$ T. Yoshie, A. Scherer, J. Hendrickson, G. Khitrova, H. M. Gibbs, G. Rupper, C. Ell, O. B. Shechekin, and D. G. Deppe, Nature (London) 432, 200-203 (2004).

${ }^{5}$ A. Badolato, K. Hennessy, M. Atature, J. Dreiser, D. Hu, P. M. Petroff, and A. Imamoglu, Science 308, 1158-1161 (2005).

${ }^{6}$ K. Hennessy, A. Badolato, M. Winger, D. Gerace, S. Gulde, S. Falt, E. L. $\mathrm{Hu}$, and A. Imamoglu, Nature (London) 445, 896-899 (2007).

${ }^{7}$ S. Strauf, Nat. Photonics 4, 132-134 (2010).

${ }^{8}$ H. Altug, D. Englund, and J. Vuckovic, Nat. Phys. 2, 484 (2006).

${ }^{9}$ S. Azzini, D. Gerace, M. Galli, I. Sagnes, R. Braive, A. Lemaître, J. Bloch, and D. Bajoni, Appl. Phys. Lett. 99, 111106 (2011).

${ }^{10}$ K. Nozaki, S. Kita, and T. Baba, Opt. Express 15, 7506-7514 (2007).

${ }^{11}$ S. Strauf, K. Hennessy, M. T. Rakher, Y. S. Choi, A. Badolato, L. C. Andreani, E. L. Hu, P. M. Petroff, and D. Bouwmeester, Phys. Rev. Lett. 96, 127404 (2006).

${ }^{12}$ T. J. Kippenberg, S. M. Spillane, and K. J. Vahala, Appl. Phys. Lett. 85, 6113 (2004).

${ }^{13}$ Y. Arakawa and H. Sakaki, Appl. Phys. Lett. 40, 939 (1982).

${ }^{14}$ E. Kapon, Proc. IEEE 80, 398 (1992).

${ }^{15}$ J. Canet-Ferrer, G. Muñoz-Matutano, D. Fuster, B. Alén, Y. González, L. González, and J. P. Martínez-Pastor, J. Appl. Phys. 110, 103502 (2011).

${ }^{16}$ B. Alén, J. Martínez-Pastor, A. García-Cristóbal, L. González, and J. M. García, Appl. Phys. Lett. 78, 4025-4027 (2001).

${ }^{17}$ M. Cao, Y. Miyake, S. Nogiwa, S. Arai, K. Furuya, and Y. Suematsu, Electron. Lett. 24, 824 (1988).

${ }^{18}$ K. A. Atlasov, M. Calic, K. F. Karlsson, P. Gallo, A. Rudra, B. Dwir, and E. Kappon, Opt. Express 17, 18178 (2009).

${ }^{19}$ L. J. Martínez, B. Alén, I. Prieto, D. Fuster, L. González, Y. González, M. L. Dotor, and P. A. Postigo, Opt. Express 17, 14993 (2009).

${ }^{20}$ M. H. Mao and H. C. Chien, Opt. Express 20, 3302 (2012).

${ }^{21}$ N. Gregersen, T. Suhr, M. Lorke, and J. Mork, Appl. Phys. Lett. 100, 131107 (2012).

${ }^{22}$ S. H. Kim, G. H. Kim, S. K. Kim, H. Y. Park, Y. H. Lee, and S. B. Kim, J. Appl. Phys. 95, 411-416 (2004).

${ }^{23}$ L. J. Martínez, I. Prieto, B. Alén, and P. A. Postigo, J. Vac. Sci. Technol. B 27, 1801-1804 (2009).

${ }^{24}$ J. Canet-Ferrer, L. J. Martínez, I. Prieto, B. Alén, G. Muñoz-Matutano, D. Fuster, Y. González, M. L. Dotor, L. González, P. A. Postigo, and J. Martínez-Pastor, Opt. Express 20, 7901-7914 (2012).

${ }^{25}$ B. Alén, D. Fuster, G. Muñoz-Matutano, J. Martínez-Pastor, Y. González, J. Canet-Ferrer, and L. González, Phys. Rev. Lett. 101, 067405 (2008).

${ }^{26}$ T. Baba and D. Sano, IEEE J. Sel. Top. Quantum Electron. 9, 1340-1346 (2003). 